Journal of Case Reports 2021;11(2):71-74

\title{
Giant Borderline Ovarian Serous Cystadenoma Weighing 10 kg
}

\author{
Federica Rigo, TizianaViora \\ Department of General and Emergency Surgery, San Giovanni Bosco Hospital, University of Turin,Turin, Italy.
}

\section{Corresponding Author: \\ Dr Federica Rigo \\ Email:federica.rigo@unito.it}

This is an Open Access article distributed under the terms of the Creative Commons Attribution License (creativecommons.org/ licenses/by/3.0).

Received : October 13, 2020

Accepted : February 9, 2021

Published : April 5,2021

\begin{abstract}
Background: Giant ovarian cystadenomas represent a severe condition, only rarely reported in literature. Usually, patients suffering of this condition undergo surgery, given the potential risk of malignancy as well as bulky symptoms. Case Report: Here we report 69 -year-old female with a rapidly-grown giant ovarian mass. As suggested by the preoperative CT-scan, bilateral ureteral stent (J-J) was placed before surgery, followed by laparotomy with hysterectomy and bilateral salpingo-oophorectomy. Histopathological examination showed that this giant mass was a borderline ovarian serous cystadenoma. During the post-operative course the patient had a minor nephrological complication related to J-J, which was resolved within 48 hours, the patient was discharged at 8 days post-surgery. Conclusion: Here we show that a multidisciplinary team might crucially help in treating this challenging condition, which usually hide unexpected pitfalls.
\end{abstract}

Keywords: Hysterectomy, Laparotomy, Ovarian Cysts, Ovarian Neoplasms, Salpingooophorectomy, Serous Cystadenoma.

\section{Introduction}

Majority of ovarian masses are detected in the early stages of gynaecological examinations making large ovarian masses rare. Other cases, on the one hand are incidentally discovered due to improved diagnostic imaging techniques [1], On the other hand, they can hugely grow up becoming symptomatic giant masses, usually associated to compression-related symptoms. The management of these masses is often related to their histopathological nature, the patient's age and the size of the mass itself. However, the adopted treatment strategy often requires surgery with a multidisciplinary approach. Ovarian tumors are classified in four major categories: epithelial tumors $(60 \%$ of all ovarian tumors and $80 \%$ of malignant ovarian tumors), germ cell tumors, sexcord-stromal tumors and metastatic tumors [2].

The concept of "borderline tumors" is known to clinical and scientific community since almost 70 years, but officially recognized by WHO and FIGO with a specific classification only 20 years after [3-5]. The term "borderline tumors" has been adopted to describe tumors associated to a greater epithelial growth than their benign counterparts, but, at the same time, not being as invasive as the malignant ones (i.e. by not invading the stroma) [6]. Recently, WHO classification of ovarian cancer updated the concept of "borderline tumors" and their role. Indeed, these tumors seem to behave diversely as well as to have different molecular signatures. Moreover they could represent a step in the progression from benign to invasive lesions $[7,8]$. Here a case of a 69 -yearold female patient with a giant borderline ovarian serous cystadenoma of weighing $10 \mathrm{~kg}$ is reported. Surprisingly, this condition has been defined as asymptomatic, despite the huge size of the mass.

\section{Case Report}

The patient is a 69-year-old woman who came into our hospital for a surgical consult. Nothing relevant was detected in her medical history, 
except cholecystectomy performed in the long past. During the first clinical visit, the patient showed a huge abdominal distension and reported to have noticed an abdominal volume increase during the last six months, without listing any particular symptom, but a slight digestive difficulty as well as infrequent constipation. An abdominal ultrasound and abdominal computer tomography (CT-scan) was performed for large abdominal distension. The CT-scan revealed a $\sim 26 \times 33 \times 20 \mathrm{~cm}$ oval cystic mass with an umbilical hernia of $\sim 8 \mathrm{~cm}$ [Fig.1]. The mass was in contiguity with the digestive structures and the anterior abdominal wall, although without any infiltration, and it seemed to be vascularized through the iliacdependent arterial circles. The performed laboratory tests were normal and tumor markers were: CEA (carcinoembryonic antigen) $5.2 \mathrm{ng} / \mathrm{mL}, \mathrm{Ca}$ 19,9 (carbohydrate antigen) $103 \mathrm{IU} / \mathrm{mL}$, AFP (alphafetoprotein) $0.8 \mathrm{IU} / \mathrm{mL}$. Due to the large volume of the mass and the pre-operative diagnostic imaging, the placement of the pre-operative bilateral J-J catheters were considered precautionary to prevent ureteral injury. A xifo-pubo incision was performed under general anesthesia, in order to bring up the lower section of the tumor originating from the right ovary. Fluid in the abdomen was observed and a sample was taken for further cytological examination. The mass developed some adherence with the jejunum mesentery and the parietal peritoneum. No secondary lesion was found on the abdominal organs. The mass was resected, followed by bilateral hysteroannessiectomy, section of the vaginal fornix and resection en bloc of the uterus as well as of the left ovary [Fig.2-4]. Afterwards, an appendectomy was performed and a plaque from the parietal peritoneum was removed and sent for extemporaneous histological examination (which, later on, showed to be negative for neoplastic cells). Moreover, there were no intra-operative complications.

The histopathology examination confirmed fibrous consistency of the plaque from the peritoneum. No anomalies were found in the

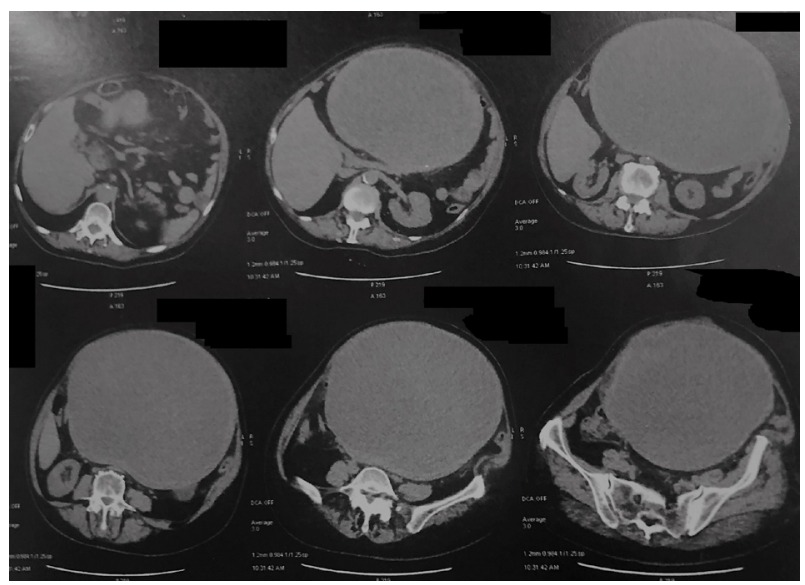

Fig.1: Pre-operative CT scan image.

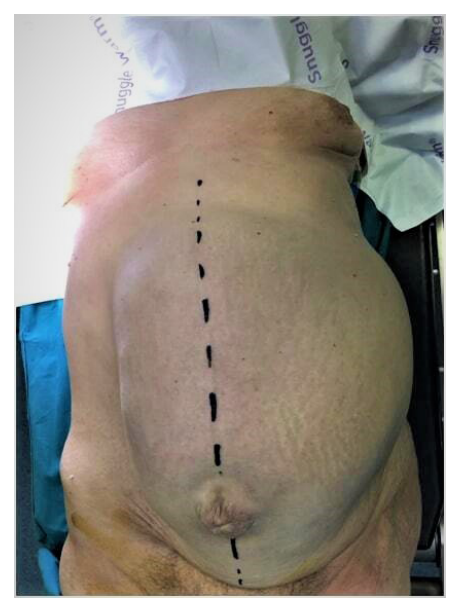

Fig.2: Huge abdominal distension of patient undergoing surgery.

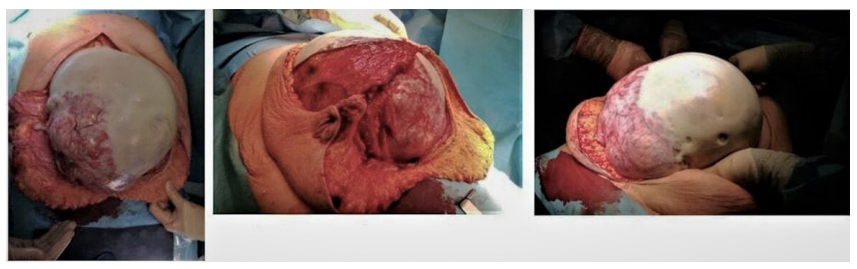

Fig.3: Mass removal during surgery.
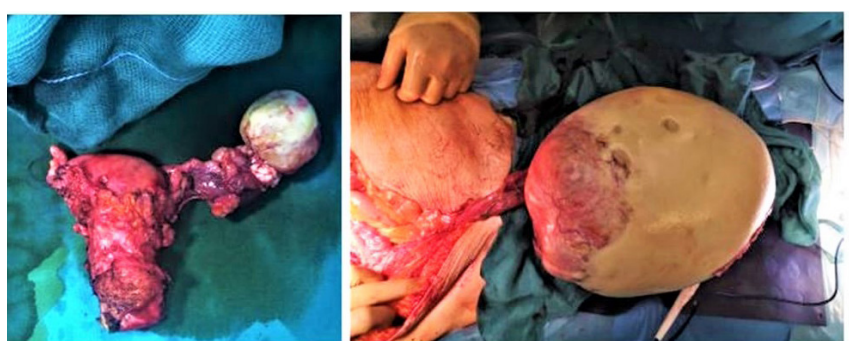

Fig.4: Left side: uterus and ovary. Right side: mass removed. 
appendix. The uterus and left ovary showed polypoid cystic atrophy. On the side of the right ovary, the giant cyst diameter was $\sim 23$ $\mathrm{cm}$ weighing $\sim 10 \mathrm{~kg}$. The interior surface of the cystic mass showed mush content and presence of solid jellis confluent deposits. The related histopathology suggested a borderline ovarian serous cystadenoma (pT1a) [Fig.5]. Following an initial regular post-operative course, urological and nephrological consulting were requested for sudden oligo-anuria and creatinine increase as shown at laboratory tests the second day after surgery. The ultrasound of the abdomen was normal without signs of hydronephrosis. Bladder catheter was replaced, and fluid infusions were increased, accordingly to urological indications. Due to the persistence of symptoms, an abdomen CT scan was required, showing a dislocation of the right J-J catheter. Following the urological consultation, the patient was moved to the operating theatre for right $\mathrm{J}-\mathrm{J}$ catheter replacement. Ensuing post-operative course was regular with a normalization of serum creatinine value, gradual resumpion of urination and subsequent removal of the bladder catheter. The patient was discharged in good condition on the eight post-operative day, with an urological indication to remove J-J catheters not earlier than 30 days from the discharge day.

\section{Discussion}

Giant ovarian cysts can be, histopathologically, either serous or mucinous and they are benign in most of the cases [9]. Such masses often require surgical treatment for compression symptoms or their potential malignant risk, albeit the occurrence of the latter seems rare. Here, the case of a 69-yearold woman, who required medical attention due to an important abdominal distension, is reported. Following a pre-operative imaging analysis, the patient underwent to mass exeresis and, notably, in this case this was possible due to multidisciplinary approach (i.e. urologists and nephrologists). The histological examination showed a borderline ovarian serous cystadenoma. The patient had a

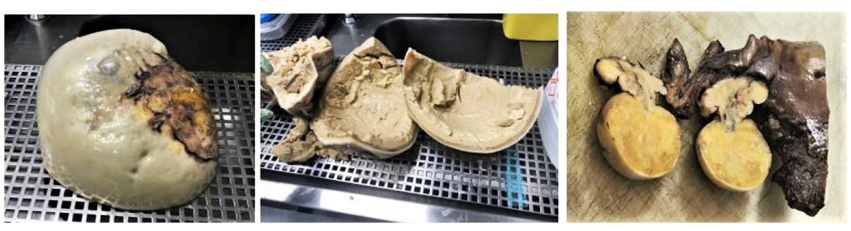

Fig.5: Histopathology examination of giant mass, uterus and left ovary.

minor nephrological complication related to J-J stents, which was resolved within 48 hours and it was discharged after eight days post-surgery. Giant ovarian mass have been rarely reported, but when it happens, multidisciplinary team might crucially help in treating this challenging condition.

\section{Conclusion}

Giant ovarian cysts, seemingly not extremely complicated to treat from a surgical point of view, they might actually hold hidden and not easily identifiable pitfalls, either intra-operatively or post-operatively. This case reports hints towards the direction that these complications can be more efficiently managed by means of a multidisciplinary strategy.

Contributors: Data collection, literature review and drafting of the article were performed by F.R. T.V. revised the article. FR will act as a study guarantor. Both authors read and approved the final version of the manuscript and are responsible for all aspects of the study.

Funding: None; Competing interests: None stated.

\section{References}

1. Brett MR, Permuth JB, Sellers TA. Epidemiology of ovarian cancer: a review. Cancer Biol Med. 2017;14:932.

2. Koonings PP, Campbell K, Mishell DR, Grimes DA. Relative frequency of primary ovarian neoplasms: a 10year review. Obstet Gynecol. 1989;74:921-926.

3. Kottmeier HL. The classification and treatment of ovarian tumours. Acta Obstet Gynecol Scand. 1952;31:313-363.

4. SerovSF, ScullyRE, SobinLHHistologictyping ofovarian tumors. World Health Organization; 1973. Available at https://apps.who.int/iris/handle/10665/41529. Accessed on October 13,2020.

5. Leppien G. Non-uterine gynecological sarcomas. Arch Gynecol Obstet. 1987;241(1):25-32. 
6. Acs G. Serous and mucinous borderline (low malignant potential) tumors of the ovary. Pathol. Patterns Rev. 2005;123:S13-S57.

7. Kurman RJ, Carcangiu ML, Herrington CS, Young RH. WHO classification of tumours of female reproductive organs $4^{\text {th }}$ edition.France; Lyon: International Agency for Research on Cancer, 2014.

8. Meinhold-Heerlein I, Fotopoulou C, Harter P, Kurzeder
C, Mustea A, Wimberger P, et al. The new WHO classification of ovarian, fallopian tube, and primary peritoneal cancer and its clinical implications. Arch Gynecol Obstet. 2016;293:695-700.

9. Alobaid A, Memon A, Alobaid S, Aldakhil L. Laparoscopic management of huge ovarian cysts. Obstetrics and Gynecology International. 2013;380854. 